\title{
Linear Isometries between Real Banach Algebras of Continuous Complex-Valued Functions
}

\author{
Davood Alimohammadi and Hadis Pazandeh \\ Department of Mathematics, Faculty of Science, Arak University, Arak 38156-8-8349, Iran \\ Correspondence should be addressed to Davood Alimohammadi; d-alimohammadi@araku.ac.ir
}

Received 27 February 2013; Revised 14 August 2013; Accepted 26 August 2013

Academic Editor: Jan Lang

Copyright (C) 2013 D. Alimohammadi and H. Pazandeh. This is an open access article distributed under the Creative Commons Attribution License, which permits unrestricted use, distribution, and reproduction in any medium, provided the original work is properly cited.

\begin{abstract}
Let $X$ and $Y$ be compact Hausdorff spaces, and let $\tau$ and $\eta$ be topological involutions on $X$ and $Y$, respectively. In 1991, Kulkarni and Arundhathi characterized linear isometries from a real uniform function algebra $A$ on $(X, \tau)$ onto a real uniform function algebra $B$ on $(Y, \eta)$ applying their Choquet boundaries and showed that these mappings are weighted composition operators. In this paper, we characterize all onto linear isometries and certain into linear isometries between $C(X, \tau)$ and $C(Y, \eta)$ applying the extreme points in the unit balls of $C(X, \tau)^{*}$ and $C(Y, \eta)^{*}$.
\end{abstract}

\section{Introduction and Preliminaries}

Let $\mathbb{R}$ and $\mathbb{C}$ denote the field of real and complex numbers, respectively. The symbol $\mathbb{F}$ denotes a field that can be either $\mathbb{R}$ or $\mathbb{C}$. The elements of $\mathbb{F}$ are called scalars. We also denote by $\mathbb{S}_{\mathbb{F}}$ the set of all $\lambda \in \mathbb{F}$ with $|\lambda|=1$.

Let $\mathfrak{X}$ be a normed space over $\mathbb{F}$. We denote by $\mathfrak{X}^{*}$ and $B_{\mathfrak{X}}$ the dual space of $\mathfrak{X}$ and the closed unit ball of $\mathfrak{X}$, respectively. For a subset $E$ of $\mathfrak{X}$, let $\operatorname{Ext}(E)$ denote the set of all extreme points of $E$. Kulkarni and Limaye showed [1, Theorem 2] that if $A$ is a nonzero linear subspace of $\mathfrak{X}$ and $\varphi \in \operatorname{Ext}\left(B_{A^{*}}\right)$, then $\varphi$ has an extension to some $\psi \in \operatorname{Ext}\left(B_{\mathfrak{X}^{*}}\right)$.

We know that if $\mathfrak{X}$ and $\mathscr{Y}$ are normed spaces over $\mathbb{F}$ and $T: \mathfrak{X} \rightarrow \mathscr{Y}$ is a linear isometry from $\mathfrak{X}$ onto $\mathscr{Y}$ over $\mathbb{F}$, then $T$ is a bijection mapping between $\operatorname{Ext}\left(B_{\mathfrak{X}}\right)$ and $\operatorname{Ext}\left(B_{\mathscr{Y}}\right)$.

Let $X$ be a compact Hausdorff space. We denote by $C_{\mathbb{F}}(X)$ the unital commutative Banach algebra of all continuous functions from $X$ into $\mathbb{F}$, with the uniform norm $\|f\|_{X}=$ $\sup \{|f(x)|: x \in X\}, f \in C_{\mathbb{F}}(X)$. We write $C(X)$ instead as $C_{\mathbb{C}}(X)$. For $x \in X$, we consider the linear functional $e_{C_{\mathbb{F}}(X), x}$ on $C_{\mathbb{F}}(X)$ defined by $e_{C_{\mathbb{F}}(X), x}(f)=f(x)\left(f \in C_{\mathbb{F}}(X)\right)$, which is called the evaluation functional on $C_{\mathbb{F}}(X)$ at $x$. Clearly, $\lambda e_{C_{\mathbb{F}}(X), x} \in B_{C_{\mathbb{F}}(X)^{*}}$ for all $(x, \lambda) \in X \times \mathbb{F}$. It is known [2, page 441] that

$$
\operatorname{Ext}\left(B_{C_{\mathbb{F}}(X)^{*}}\right)=\left\{\lambda e_{C_{\mathbb{F}}(X), x}:(x, \lambda) \in X \times \mathbb{S}_{\mathbb{F}}\right\} .
$$

Let $A$ be a real or complex linear subspace of $C(X)$. A nonempty subset $S$ of $X$ is called a boundary for $A$ (with respect to $X$ ), if for each $f \in A$ the function $|f|$ assumes its maximum on $X$ at some $x \in S$. We denote by $\Gamma(A, X)$ the intersection of all closed boundaries for $A$. If $\Gamma(A, X)$ is a boundary for $A$, it is called the Shilov boundary for $A$ (with respect to $X$ ).

Let $A$ be linear subspace of $C_{\mathbb{F}}(X)$ containing $1_{X}$, the constant function with value 1 on $X$. A representing measure for $\varphi \in A^{*}$ is an $\mathbb{F}$-valued regular Borel measure $\mu$ on $X$ such that $\varphi(f)=\int_{X} f d \mu$ for all $f \in A$. Let $K_{\mathbb{F}}(A)=\{\varphi \in$ $\left.A^{*}:\|\varphi\|=\varphi\left(1_{X}\right)=1\right\}$. If $x \in X$, then $\left.e_{C_{F}(X), x}\right|_{A} \in K_{\mathbb{F}}(A)$ and $\delta_{x}$, the point mass measure on $X$ at $x$, is a representing measure for $\left.e_{C_{F}(X), x}\right|_{A}$. We denote by $\operatorname{Ch}(A, X)$ the set of all $x \in X$ for which $\delta_{x}$ is the only representing measure for $\left.e_{C_{F}(X), x}\right|_{A}$. If $\operatorname{Ch}(A, X)$ is a boundary for $A$, it is called the Choquet boundary for $A$ (with respect to $X$ ). We know that

$$
\Gamma\left(C_{\mathbb{F}}(X), X\right)=\operatorname{Ch}\left(C_{\mathbb{F}}(X), X\right)=X .
$$

Let $A$ be a real linear subspace of $C(X)$, and let $S$ be nonempty subset of $X$. We say that $A$ is extremely regular at $S$ if for every open neighborhood $U$ of $S$ and for each $\varepsilon>0$ there is a function $f \in A$ with $\|f\|_{X}=1$ such that $f(x)=1$ for all $x \in S$ and $|f(y)|<1$ for all $y \in X \backslash U$. 
Let $X$ be a nonempty set. A self-map $\tau: X \rightarrow X$ is called an involution on $X$ if $\tau(\tau(x))=x$ for all $x \in X$. A subset $S$ of $X$ is called $\tau$-invariant if $\tau(S) \subseteq S$. Clearly, if $S \subseteq X$ is $\tau$-invariant, then $\tau(S)=S$. A $\tau$-invariant measure on $E$ is a measure $\mu$ on $E$ such that $\mu \circ \tau=\mu$.

Let $X$ be a topological space. An involution $\tau$ on $X$ is called a topological involution on $X$, if $\tau$ is continuous.

Let $X$ be a compact Hausdorff space, and let $\tau$ be a topological involution on $X$. We define

$$
C(X, \tau)=\{f \in C(X): f \circ \tau=\bar{f}\}
$$

Then, $C(X, \tau)$ is a unital uniformly closed self-adjoint real subalgebra of $C(X)$ which separates the points of $X$ and does not contain $i_{X}$, the constant function with value $i$ on $X$. Note that $\operatorname{Re} f \in C(X, \tau)$ for all $f \in C(X, \tau)$. Moreover, $C(X)=$ $C(X, \tau) \oplus i C(X, \tau)$ and

$$
\max \left\{\|f\|_{X},\|g\|_{X}\right\} \leq\|f+i g\|_{X} \leq 2 \max \left\{\|f\|_{X},\|g\|_{X}\right\},
$$

for all $f, g \in C(X, \tau)$. In fact, the complex Banach algebra $\left(C(X),\|\cdot\|_{X}\right)$ can be regarded as the complexification of the real Banach algebra $\left(C(X, \tau),\|\cdot\|_{X}\right)$. Note that $C(X, \tau)=$ $C_{\mathbb{R}}(X)$ if and only if $\tau$ is the identity self-map on $X$. Hence, the class of real Banach algebras $C(X, \tau)$ is, in fact, larger than the class of real Banach algebras $C_{\mathbb{R}}(X)$.

Let $X$ be a compact Hausdorff space, $\tau$ a topological involution on $X$, and $E$ a subset of $C(X, \tau)$. We say that $E$ separates the points of $X / \tau$ if for each $x, y \in X$ with $y \notin$ $\{x, \tau(x)\}$ there exists a function $f$ in $E$ such that $f(x) \neq f(y)$. It is known [3, Lemma 1.3.7] that if $\operatorname{Re} C(X, \tau)=\{\operatorname{Re} f: f \in$ $C(X, \tau)\}$, then $\operatorname{Re} C(X, \tau)$ separates the points of $X / \tau$.

Let $X$ be a compact Hausdorff space. We assume that $C(X)_{r}$ denotes $C(X)$, regarded as a real normed space by restricting the scalar multiplication to real scalars. Let $\tau$ be the topological involution on $X \times\{0,1\}$ defined by $\tau(x, 0)=(x, 1)$ $(x \in X)$ and $\tau(x, 1)=(x, 0)(x \in X)$. Then the map $\Psi: C(X)_{r} \rightarrow C(X \times\{0,1\}, \tau)$ defined by

$$
\begin{array}{r}
(\Psi f)(x, 0)=f(x), \quad(\Psi f)(x, 1)=\overline{f(x)} \\
\left(f \in C(X)_{r} ; x \in X\right),
\end{array}
$$

is an isometrical isomorphism from $C(X)_{r}$ onto $C(X \times$ $\{0,1\}, \tau)$. Hence, the class of real Banach algebras $C(Y, \tau)$ is, in fact, larger than the class of real Banach algebras $C(X)_{r}$.

Let $X$ be a compact Hausdorff space, and let $\tau$ be a topological involution on $X$. Let $A$ be a real subspace of $C(X, \tau)$ containing $1_{X}$. A real part representing measure for $\varphi \in A^{*}$ is a $\tau$-invariant regular real Borel measure $\mu$ on $X$ such that $\varphi(f)=\int_{X} \operatorname{Re} f d \mu$ for all $f \in A$. Let $K_{\mathbb{R}}(A)=\left\{\varphi \in A^{*}\right.$ : $\left.\|\varphi\|=\varphi\left(1_{X}\right)=1\right\}$. If $x \in X$, then $\operatorname{Re}\left(e_{C(X),\left.x\right|_{A}}\right) \in K_{\mathbb{R}}(A)$ and the measure $m_{x}=(1 / 2)\left(\delta_{x}+\delta_{\tau(x)}\right)$ is a real part representing measure for $\operatorname{Re}\left(\left.e_{C(X), x}\right|_{A}\right)$. We denote by $\operatorname{Ch}(A, X, \tau)$ the set of all $x \in X$ for which $m_{x}$ is the only real part representing measure for $\operatorname{Re}\left(e_{C(X), x}||_{A}\right)$. Clearly, $\operatorname{Ch}(A, X, \tau)$ is a $\tau$-invariant subset of $X$. If $\operatorname{Ch}(A, X, \tau)$ is a boundary for $A$, it is called the Choquet boundary for $A$ with respect to $(X, \tau)$. We know that $\operatorname{Ch}(C(X, \tau), X, \tau)=X$.
The real Banach algebra $C(X, \tau)$ and its real linear subspaces were first considered by Kulkarni and Limaye in [4]. For a detailed account of several properties of $C(X, \tau)$, we refer to [3].

Let $X$ be a compact Hausdorff space. For each $(x, \lambda) \in$ $X \times \mathbb{C}$, define the map $\psi_{C(X), x, \lambda}: C(X)_{r} \rightarrow \mathbb{R}$ by

$$
\psi_{C(X), x, \lambda}(f)=\operatorname{Re}(\lambda f(x)), \quad\left(f \in C(X)_{r}\right),
$$

in fact, $\psi_{C(X), x, \lambda}=\operatorname{Re}\left(\lambda e_{C(X), x}\right)$. Clearly, $\psi_{C(X), x, \lambda} \in B_{\left(C(X)_{r}\right)^{*}}$.

Let $\tau$ be a topological involution on $X$. For each $(x, \lambda) \epsilon$ $X \times \mathbb{C}$ define the map $\varphi_{C(X, \tau), x, \lambda}: C(X, \tau) \rightarrow \mathbb{R}$ by $\varphi_{C(X, \tau), x, \lambda}=\left.\psi_{C(X), x, \lambda}\right|_{C(X, \tau)}$. Kulkarni and Limaye showed $[1$, Proposition 3] that

$$
\operatorname{Ext}\left(B_{\left(C(X)_{r}\right)^{*}}\right)=\left\{\psi_{C(X), x, \lambda}:(x, \lambda) \in X \times S_{\mathbb{C}}\right\} .
$$

Applying this result, they obtained the following theorem.

Theorem 1 (see [1, Corollary 5]). Let X be a compact Hausdorff space, and let $\tau$ be a topological involution on X. Suppose that

$$
\begin{aligned}
P_{X, \tau}= & \left\{(x, \lambda) \in X \times S_{\mathbb{C}}: \tau(x) \neq x\right\} \\
& \cup\left\{(x, \lambda) \in X \times S_{\mathbb{R}}: \tau(x)=x\right\} .
\end{aligned}
$$

Then

$$
\operatorname{Ext}\left(B_{C(X, \tau)^{*}}\right)=\left\{\varphi_{C(X, \tau), x, \lambda}:(x, \lambda) \in P_{X, \tau}\right\} .
$$

The classical Banach-Stone theorem states that if $T$ is a linear isometry from $C_{\mathbb{F}}(X)$ onto $C_{\mathbb{F}}(Y)$, then there exists a homeomorphism $h$ from $Y$ onto $X$ and a continuous function $a \in C_{\mathbb{F}}(Y)$ with $a(Y) \subseteq \mathbb{S}_{\mathbb{F}}$ such that

$$
(T f)(y)=a(y) f(h(y)), \quad\left(f \in C_{\mathbb{F}}(X), y \in Y\right) .
$$

This well-known theorem has been generalized in several directions. In 1966, an important generalization was given by Holsztyński [5] by considering into linear isometries as the following.

Theorem 2. Let $X$ and $Y$ be compact Hausdorff spaces. If $T$ is a linear isometry from $C_{\mathbb{F}}(X)$ into $C_{\mathbb{F}}(Y)$, then there exists a closed subset $Y_{0}$ of $Y$, a continuous map $\varphi$ from $Y_{0}$ onto $X$ and a function $a \in C_{\mathbb{F}}(Y)$ with $\|a\|_{Y}=1$ and $a\left(Y_{0}\right) \subseteq \mathbb{S}_{\mathbb{F}}$ such that

$$
(T f)(y)=a(y) f(h(y)), \quad \forall f \in C_{\mathbb{F}}(X), \forall y \in Y_{0} .
$$

In 1975, another important generalization of BanachStone theorem was given by Novinger [6] for certain complex subalgebras of $C(X)$ and $C(Y)$ considering the concept of their Choquet boundaries. In 1991, Kulkarni and Arundhathi [7] generalized the given results by Novinger for certain real subalgebras of $C(X, \tau)$ and $C(Y, \eta)$ considering the concept of their Choquet boundaries. We can cite some other generalizations of Banach-Stone theorem, for example, the generalization obtained by Cambern [8] for space of vector-valued continuous functions, by Jeang and Wong [9] for spaces of scalar-valued continuous functions vanishing 
at infinity, by Araujo and Font [10] for certain subspaces of scalar-valued continuous functions considering concepts of their Choquet and Shilov boundaries, and by JiménezVargas and Villegas-Vallecillos [11] for spaces of scalar-valued Lipschitz functions.

Throughout the rest of this paper, $X$ and $Y$ are compact Hausdorff spaces and $\tau$ and $\eta$ are topological involutions on $X$ and $Y$, respectively.

In this paper, we characterize all linear isometries from $C(X, \tau)$ onto $C(Y, \eta)$ and certain linear isometries from $C(X, \tau)$ into $C(Y, \eta)$, applying the extreme points in $B_{C(X, \tau)^{*}}$ and $B_{C(Y, \eta)^{*}}$. Our results in Section 2 are some of the given results by Kulkarni and Arundhathi in [7] that we obtain by applying the extreme points in $B_{C(X, \tau)^{*}}$ and $B_{C(Y, \eta)^{*}}$. The main result in Section 3 is a generalization of the given result by Holsztyński in [5] for certain into isometries.

\section{Onto Linear Isometries}

We first determine unit-preserving linear isometries from $C(X, \tau)$ onto $C(Y, \eta)$. For this purpose, we need the following lemmas.

Lemma 3 (see [3, Lemma 5.1.1]). Let $A$ and $B$ be real linear subspaces of $C(X, \tau)$ and $C(Y, \eta)$, respectively. Suppose that $x \in$ $X, y \in Y, A$ is extremely regular at $\{x, \tau(x)\}$ and $B$ is extremely regular at $\{y, \eta(y)\}$. Let $T: A \rightarrow B$ be a linear isometry from $A$ onto $B$ and $\operatorname{Re}((T f)(y))=\operatorname{Re}(f(x))$ for all $f \in A$. Then, $(T f)(y)=f(x)$ for all $f \in A$ or $(T f)(y)=f(\tau(x))$ for all $f \in A$.

Lemma 4. Let $S$ be a nonempty $\tau$-invariant closed subset of $X$. Then, $C(X, \tau)$ is extremely regular at $S$. In particular, $C(X, \tau)$ is extremely regular at $\{x, \tau(x)\}$ for all $x$ in $X$.

Proof. Assume that $\varepsilon>0$ and $U$ is an open neighborhood of $S$ in $X$. By Urysohn's lemma, there exists a function $g \in C(X)$ with $0 \leq g(x) \leq 1$ for all $x \in X, g(x)=1$ for all $x \in S$, and $g(x)=0$ for all $x \in X \backslash U$. Define the function $f: X \rightarrow \mathbb{C}$ by $f=(g \circ \tau) g$. Then, $f \in C(X, \tau),\|f\|_{X}=1, f(x)=1$ for all $x \in S$ and $|f(x)|<\varepsilon$ for all $x \in X \backslash U$. Hence, $C(X, \tau)$ is extremely regular at $S$.

Theorem 5. Let $T: C(X, \tau) \rightarrow C(Y, \eta)$ be a linear isometry from $C(X, \tau)$ onto $C(Y, \eta)$ with $T 1_{X}=1_{Y}$. Then, there exists a homeomorphism $h$ from $Y$ onto $X$ with $h \circ \eta=\tau \circ h$ on $Y$ such that

$$
(T f)(y)=f(h(y)), \quad \forall f \in C(X, \tau), \forall y \in Y .
$$

Proof. We claim that

$$
Y=\left\{y \in Y: T^{*}\left(\varphi_{C(Y, \eta), y, 1}\right) \in \operatorname{Ext}\left(B_{C(X, \tau)^{*}}\right)\right\} .
$$

Let $y \in Y$. By Theorem $1, \varphi_{C(Y, \eta), y, 1} \in \operatorname{Ext}\left(B_{C(Y, \eta)^{*}}\right)$. Since $T$ is a linear isometry from $C(X, \tau)$ onto $C(Y, \eta)$, we conclude that $T^{*}$ is a linear isometry from $C(Y, \eta)^{*}$ onto $C(X, \tau)^{*}$ and so

$$
T^{*}\left(\operatorname{Ext}\left(B_{C(Y, \eta)^{*}}\right)\right)=\operatorname{Ext}\left(B_{C(X, \tau)^{*}}\right) .
$$

Therefore, $T^{*}\left(\varphi_{C(Y, \eta), y, 1}\right) \in \operatorname{Ext}\left(B_{C(X, \tau)^{*}}\right)$. Hence, our claim is justified.

We now show that for each $y \in Y$ there exists a unique $x \in X$ such that

$$
(T f)(y)=f(x), \quad \forall f \in C(X, \tau) .
$$

Let $y \in Y$. By $(13)$, we have $T^{*}\left(\varphi_{C(Y, \eta), y, 1}\right) \in \operatorname{Ext}\left(B_{C(X, \tau)^{*}}\right)$. Hence, by Theorem 1 , there exists $\left(x_{0}, \lambda_{0}\right) \in X \times S_{\mathbb{C}}$ such that

$$
T^{*}\left(\varphi_{C(Y, \eta), y, 1}\right)=\varphi_{C(X, \tau), x_{0}, \lambda_{0}} .
$$

Since $1_{X} \in C(X, \tau)$ and $T 1_{X}=1_{Y}$, by $(16)$, we have

$$
\begin{aligned}
\operatorname{Re}\left(\lambda_{0}\right) & =\operatorname{Re}\left(\lambda_{0} 1_{X}\left(x_{0}\right)\right)=\varphi_{C(X, \tau), x_{0}, \lambda_{0}}\left(1_{X}\right) \\
& =T^{*}\left(\varphi_{C(Y, \eta), y, 1}\right)\left(1_{X}\right)=\varphi_{C(Y, \eta), y, 1}\left(T 1_{X}\right) \\
& =\varphi_{C(Y, \eta), y, 1}\left(1_{Y}\right)=\operatorname{Re}\left(1_{Y}(y) e_{C(Y), y}\left(1_{Y}\right)\right) \\
& =\operatorname{Re}\left(1_{Y}(y) 1_{Y}(y)\right)=\operatorname{Re}(1) \\
& =1 .
\end{aligned}
$$

Since $\left|\lambda_{0}\right|=1$ and $\operatorname{Re}\left(\lambda_{0}\right)=1$, we have $\operatorname{Im}\left(\lambda_{0}\right)=0$ and so $\lambda_{0}=1$. Thus, by (16), we have

$$
T^{*}\left(\varphi_{C(Y, \eta), y, 1}\right)=\varphi_{C(X, \tau), x_{0}, 1} .
$$

From (18) we deduce that

$$
\operatorname{Re}((T f)(y))=\operatorname{Re}\left(f\left(x_{0}\right)\right), \quad \forall f \in C(X, \tau) .
$$

Since $T$ is a linear isometry from $C(X, \tau)$ onto $C(Y, \eta)$ and by Lemma $4, C(X, \tau)$ is extremely regular at $\left\{x_{0}, \tau\left(x_{0}\right)\right\}$, and $C(Y, \eta)$ is extremely regular at $\{y, \eta(y)\}$, we conclude that $(T f)(y)=f\left(x_{0}\right)$ for all $f$ in $C(X, \tau)$ or $(T f)(y)=f\left(\tau\left(x_{0}\right)\right)$ for all $f \in C(X, \tau)$ by Lemma 3 . We assume that $x=x_{0}$ whenever $(T f)(y)=f\left(x_{0}\right)$ for all $f$ in $C(X, \tau)$ and $x=\tau\left(x_{0}\right)$ whenever $(T f)(y)=f\left(\tau\left(x_{0}\right)\right)$ for all $f$ in $C(X, \tau)$. Then, $x \in X$ and we have

$$
(T f)(y)=f(x), \quad \forall f \in C(X, \tau) .
$$

This proves the existent of $x$. To show uniqueness, assume that there exists $x^{\prime} \in X$ such that

$$
(T f)(y)=f\left(x^{\prime}\right), \quad \forall f \in C(X, \tau) .
$$

Then, $f(x)=f\left(x^{\prime}\right)$ for all $f$ in $C(X, \tau)$. This implies that $x=$ $x^{\prime}$ since $C(X, \tau)$ separates the points of $X$.

Now we define the map $h: Y \rightarrow X$ by $h(y)=x$ whenever $(T f)(y)=f(x)$ for all $f$ in $C(X, \tau)$. In fact, we have

$$
(T f)(y)=f(h(y)), \quad \forall f \in C(X, \tau) \quad \forall y \in Y .
$$

Now we prove that $h \circ \eta=\tau \circ h$ on $Y$. Let $y \in Y$. Then, $\eta(y) \in Y$. From (22), for each $f \in C(X, \tau)$ we have

$$
\begin{aligned}
f((h \circ \eta)(y)) & =f(h(\eta(y)))=(T f)(\eta(y)) \\
& =\overline{(T f)(y)}=\overline{f(h(y))} \\
& =f(\tau(h(y)))=f((\tau \circ h)(y)) .
\end{aligned}
$$


This implies that $(h \circ \eta)(y)=(\tau \circ h)(y)$ since $C(X, \tau)$ separates the points of $X$. Therefore, $h \circ \eta=\tau \circ h$ on $Y$.

Continually, we prove that $h$ is bijective. Since $T^{-1}$ is a linear isometry from $C(Y, \eta)$ onto $C(X, \tau)$ with $T^{-1} 1_{Y}=1_{X}$, by the above argument, we deduce that there exists a map $k: X \rightarrow Y$ such that

$$
\left(T^{-1} g\right)(x)=g(k(x)), \quad \forall g \in C(Y, \eta), \forall x \in X .
$$

To prove the injectivity of $h$, let $y \in Y$. For each $g \in C(Y, \eta)$, by (22) and (24) we have

$$
\begin{aligned}
g((k \circ h)(y)) & =g(k(h(y)))=\left(T^{-1} g\right)(h(y)) \\
& =\left(T\left(T^{-1} g\right)\right)(y)=g(y) .
\end{aligned}
$$

This implies that $(k \circ h)(y)=y$ since $C(Y, \eta)$ separates the points of $Y$. Therefore, $k \circ h=I_{Y}$, the identity mapping on $Y$, and so $h$ is injective.

To prove that $h$ is onto, let $x \in X$. For each $f \in C(X, \tau)$, by (24) and (22) we have

$$
\begin{aligned}
f((h \circ k)(x)) & =f(h(k(x)))=(T f)(k(x)) \\
& =\left(T^{-1}(T f)\right)(x)=f x .
\end{aligned}
$$

This implies that $(h \circ k)(x)=x$ since $C(X, \tau)$ separates the points of $X$. Therefore $k \circ h=I_{X}$, the identity mapping on $X$, and so $h$ is onto.

We now check that $h: Y \rightarrow X$ is continuous. Let $y \in Y$, and let $\left\{y_{\alpha}\right\}_{\alpha \in J}$ be a net in $Y$ such that $\lim _{\alpha} y_{\alpha}=y$ in $Y$. Then we have

$$
\lim _{\alpha}(T f)\left(y_{\alpha}\right)=(T f)(y), \quad \forall f \in C(X, \tau) .
$$

By (27) and definition of $h$, we deduce that

$$
\lim _{\alpha} f\left(h\left(y_{\alpha}\right)\right)=f(h(y)), \quad \forall f \in C(X, \tau) .
$$

Since $C(X, \tau)$ separates the points of $X$, by (28), we have $\lim _{\alpha} h\left(y_{\alpha}\right)=h(y)$ in $X$. Therefore, $h: Y \rightarrow X$ is continuous.

Since $h: Y \rightarrow X$ is a bijective continuous mapping, $Y$ is a compact space, and $X$ is a Hausdorff space, we conclude that $h^{-1}: X \rightarrow Y$ is continuous, and so $h$ is a homeomorphism. Hence, the proof is complete.

Corollary 6. Let $T: C(X, \tau) \rightarrow C(Y, \eta)$ be an isometry mapping from $C(X, \tau)$ onto $C(Y, \eta)$ with $T(0)=0$ and $T 1_{X}=$ $1_{Y}$. Then $T$ is an isomorphism.

Proof. Since $\left(C(X, \tau),\|\cdot\|_{X}\right)$ and $\left(C(Y, \eta),\|\cdot\|_{Y}\right)$ are real Banach algebras and $T$ is an isometry from $C(X, \tau)$ onto $C(Y, \eta)$ with $T(0)=0$, we conclude that $T$ is a linear isometry by Mazur-Ulam theorem (see [12]). Since $T 1_{X}=1_{Y}$, applying Theorem 5, we deduce that there exists a homeomorphism $h$ from $Y$ onto $X$ such that

$$
(T f)(y)=f(h(y)), \quad \forall f \in C(X, \tau), \forall y \in Y .
$$

It follows that $T$ is a homomorphism. Hence, the proof is complete. ing).

We now study the onto case (not necessarily unit preserv-

Lemma 7. Let $f \in C(X, \tau)$ with $\|f\|_{X}=1$. Then, $|f(x)|=1$ for all $x \in X$, if for every $\varepsilon>0$ there exists $\delta>0$ such that $g \in C(x, \tau)$ with $\|g\|_{X} \geq \varepsilon$ implies that

$$
\max \left\{\|f+g\|_{X},\|f-g\|_{X}\right\} \geq 1+\delta .
$$

Proof. Assume that there exists $x_{0} \in X$ such that $\left|f\left(x_{0}\right)\right| \neq 1$. Then, $\left|f\left(x_{0}\right)\right|<1$ since $\|f\|_{X}=1$. Let $\varepsilon=(1 / 3)\left(1-\left|f\left(x_{0}\right)\right|\right)$. Then, $0<\varepsilon<1 / 3$ and $\left|f\left(x_{0}\right)\right|<1-2 \varepsilon$. Take $V=\{x \in X$ : $|f(x)|<1-\varepsilon\}$. Since $f \in C(X, \tau)$, we deduce that $V$ is a $\tau$ invariant open subset of $X$ and $\left\{x_{0}, \tau\left(x_{0}\right)\right\} \subseteq V$. By Urysohn's lemma, there exists $g_{1} \in C(X)$ such that $0 \leq g_{1}(x) \leq 1$ for all $x \in X, g_{1}\left(x_{0}\right)=g_{1}\left(\tau\left(x_{0}\right)\right)=1$, and $g_{1}(x)=0$ for all $x \in X \backslash V$. Let $g=\varepsilon g_{1}\left(g_{1} \circ \tau\right)$. Then, $g \in C(X, \tau), 0 \leq g(x) \leq \varepsilon$ for all $x \in X, g\left(x_{0}\right)=g\left(\tau\left(x_{0}\right)\right)=\varepsilon$, and $g(x)=0$ for all $x \in X \backslash V$. Therefore,

$$
\|f+g\|_{X} \leq 1, \quad\|f-g\|_{X} \leq 1 .
$$

Hence, for each $\delta>0$ we have

$$
\max \left\{\|f+g\|_{X},\|f-g\|_{Y}\right\}<1+\delta .
$$

This completes the proof.

Lemma 8. Let $T: C(X, \tau) \rightarrow C(Y, \eta)$ be a linear isometry from $C(X, \tau)$ onto $C(Y, \eta)$, and let $a=T 1_{X}$. Then, $|a(y)|=1$ for all $y \in Y$.

Proof. Since $a \in C(Y, \eta)$ and $\|a\|_{Y}=\left\|T 1_{X}\right\|_{Y}=\left\|1_{X}\right\|_{X}=1$, by Lemma 7, it is sufficient to show that for each $\varepsilon>0$ there exists $\delta>0$ such that $g \in C(Y, \eta)$ with $\|g\|_{Y} \geq \varepsilon$ implies that

$$
\max \left\{\|a+g\|_{Y},\|a-g\|_{X}\right\} \geq 1+\delta .
$$

Let $\varepsilon>0$, and let $g \in C(Y, \eta)$ with $\|g\|_{Y} \geq \varepsilon$. The surjectivity of $T$ implies that there exists $f \in C(X, \tau)$ such that $T f=g$. Since $T$ is an isometry, we have $\|f\|_{X}=\|g\|_{Y} \geq \varepsilon$. This implies that there exists $x_{0} \in X$ such that $\left|f\left(x_{0}\right)\right| \geq \varepsilon$. Therefore,

$$
\begin{aligned}
\max & \left\{\left|1+f\left(x_{0}\right)\right|^{2},\left|1-f\left(x_{0}\right)\right|^{2}\right\} \\
& \geq \frac{1}{2}\left[\left|1+f\left(x_{0}\right)\right|^{2}+\left|1-f\left(x_{0}\right)\right|^{2}\right] \\
& =1+\left|f\left(x_{0}\right)\right|^{2} \\
& \geq 1+\varepsilon^{2} .
\end{aligned}
$$

It follows that

$$
\max \left\{\left|1+f\left(x_{0}\right)\right|,\left|1-f\left(x_{0}\right)\right|\right\} \geq \sqrt{1+\varepsilon^{2}} .
$$

Hence,

$$
\max \left\{\left\|1_{X}+f\right\|_{X},\left\|1_{X}-f\right\|_{X}\right\} \geq \sqrt{1+\varepsilon^{2}} .
$$

Since $\|a+g\|_{Y}=\left\|1_{X}+f\right\|_{X}$ and $\|a-g\|_{Y}=\left\|1_{X}-f\right\|_{X}$, we have

$$
\max \left\{\|a+g\|_{Y},\|a-g\|_{Y}\right\} \geq \sqrt{1+\varepsilon^{2}} .
$$

It is enough that we choose $\delta=\sqrt{1+\varepsilon^{2}}-1$. 
In the following result, we show that every linear isometry from $C(X, \tau)$ onto $C(Y, \eta)$ is a weighted composition operator.

Theorem 9. Let $T: C(X, \tau) \rightarrow C(Y, \eta)$ be a linear isometry from $C(X, \tau)$ onto $C(Y, \eta)$. Then, there exist a function a $\epsilon$ $C(Y, \eta)$ with $|a(y)|=1$ for all $y \in Y$ and a homeomorphism $h$ from $Y$ onto $X$ with $h \circ \eta=\tau \circ h$ on $Y$ such that

$$
(T f)(y)=a(y) f(h(y)), \quad \forall f \in C(X, \tau), \forall y \in Y .
$$

Proof. Assume that $a=T 1_{X}$. Then $a \in C(Y, \eta)$. By Lemma 8, we have $|a(y)|=1$ for all $y \in Y$. Clearly, $(\bar{a})(T f) \in C(Y, \eta)$ for all $f \in C(X, \tau)$ and $(\bar{a})\left(T 1_{X}\right)=1_{Y}$. We define the map $\bar{a} \cdot T: C(X, \tau) \rightarrow C(Y, \eta)$ by $(\bar{a} \cdot T)(f)=(\bar{a})(T f)$. Then, $\bar{a} \cdot T$ is a linear isometry from $C(X, \tau)$ onto $C(Y, \eta)$, and $(\bar{a} \cdot T)\left(1_{X}\right)=$ $1_{Y}$. By Theorem 5 , there exists a homeomorphism $h$ from $Y$ onto $X$ with $h \circ \eta=\tau \circ h$ on $Y$ such that

$$
((\bar{a} \cdot T)(f))(y)=f(h(y)), \quad \forall f \in C(X, \tau), \forall y \in Y .
$$

This implies that

$$
(T f)(y)=a(y) f(h(y)), \quad \forall f \in C(X, \tau), \forall y \in Y .
$$

Hence, the proof is complete.

\section{Into Linear Isometries}

We formulate our main result in this section which is a version for into linear isometries of $C(X, \tau)$-spaces of a known Holsztyńskis's theorem (Theorem 2) for into linear isometries of $C_{\mathbb{F}}(X)$-spaces. We first study unit-preserving linear isometries from $C(X, \tau)$ into $C(Y, \eta)$.

Theorem 10. Let $T: C(X, \tau) \rightarrow C(Y, \eta)$ be a linear isometry from $C(X, \tau)$ into $C(Y, \eta)$ satisfying the following conditions:

(i) $T 1_{X}=1_{Y}$,

(ii) if $y \in Y$ and there is a point $x \in X$ such that $\operatorname{Re}((T f)(y))=\operatorname{Re}(f(x))$ for all $f \in C(X, \tau)$, then $T(C(X, \tau))$ is extremely regular at $\{y, \tau(y)\}$.

Then, there exist a $\eta$-invariant closed boundary $Y_{0}$ for $T(C(X, \tau))$ and a continuous map $h$ from $Y_{0}$ onto $X$ with $h \circ \eta=\tau \circ h$ on $Y_{0}$ such that

$$
(T f)(y)=f(h(y)), \quad \forall f \in C(X, \tau), \forall y \in Y_{0} .
$$

Proof. Let $E=T(C(X, \tau))$. Since $T: C(X, \tau) \rightarrow C(Y, \eta)$ is a linear isometry, we deduce that $E$ is a uniformly closed linear subspace of $C(Y, \eta)$. Moreover, $T\left(1_{X}\right)=1_{Y}$ implies that $1_{Y} \in$ $E$. We define the map $S: C(X, \tau) \rightarrow E$ by $S f=T f$. Then, $S$ is a linear isometry from the real Banach space $\left(C(X, \tau),\|\cdot\|_{X}\right)$ onto the real Banach space $\left(E,\|\cdot\|_{Y}\right)$. Therefore, $S^{*}$ is a linear isometry from $E^{*}$ onto $C(X, \tau)^{*}$ and so

$$
S^{*}\left(\operatorname{Ext}\left(B_{E^{*}}\right)\right)=\operatorname{Ext}\left(B_{C(X, \tau)^{*}}\right) .
$$

Now we define

$$
Y_{0}=\left\{y \in Y: S^{*}\left(\left.\varphi_{C(Y, \eta), y, 1}\right|_{E}\right) \in \operatorname{Ext}\left(B_{C(X, \tau)^{*}}\right)\right\} .
$$

We first show that $Y_{0}$ is nonempty. To prove this fact, we show that the following statement, namely (I), holds.

(I) For each $x \in X$ there exists $y_{0} \in Y$ such that $S^{*}\left(\left.\varphi_{C(Y, \eta), y, 1}\right|_{E}\right)=\varphi_{C(X, \tau), x, 1}$.

Let $x \in X$. Then $\varphi_{C(X, \tau), x, 1} \in \operatorname{Ext}\left(B_{C(X, \tau)^{*}}\right)$ by Theorem 1 . From (42), there exists $\Lambda \in \operatorname{Ext}\left(B_{E^{*}}\right)$ such that

$$
S^{*} \Lambda=\varphi_{C(X, \tau), x, 1} .
$$

Since $E$ is a linear subspace of $C(Y, \eta)$, there exists $\widetilde{\Lambda} \epsilon$ $\operatorname{Ext}\left(B_{C(Y, \eta)^{*}}\right)$ such that

$$
\left.\widetilde{\Lambda}\right|_{E}=\Lambda
$$

By Theorem 1 , there exists $\left(y_{0}, \gamma_{0}\right) \in Y \times \mathbb{S}_{\mathbb{C}}$ such that

$$
\widetilde{\Lambda}=\varphi_{C(X), y_{0}, \gamma_{0}} .
$$

From (44), (45), and (46), we have

$$
S^{*}\left(\left.\varphi_{C(Y, \eta), y_{0}, \gamma_{0}}\right|_{E}\right)=\varphi_{C(X, \tau), x, 1} .
$$

Since $1_{X} \in C(X, \tau)$ and $T 1_{X}=1_{Y}$, by (47) we have

$$
\begin{aligned}
\operatorname{Re}\left(\gamma_{0}\right) & =\operatorname{Re}\left(\gamma_{0} 1_{Y}\left(y_{0}\right)\right)=\varphi_{C(Y, \eta), y_{0}, \gamma_{0}}\left(1_{Y}\right) \\
& =\varphi_{C(Y, \eta), y_{0}, \gamma_{0}}\left(T 1_{X}\right)=\varphi_{C(Y, \eta), y_{0}, \gamma_{0}}\left(S 1_{X}\right) \\
& =S^{*}\left(\left.\varphi_{C(Y, \eta), y_{0}, \gamma_{0}}\right|_{E}\right)\left(1_{X}\right)=\varphi_{C(X, \tau), x, 1}\left(1_{X}\right) \\
& =\operatorname{Re}\left(1 e_{C(X), x}\left(1_{X}\right)\right)=\operatorname{Re}\left(1_{X}(x)\right) \\
& =\operatorname{Re}(1)=1 .
\end{aligned}
$$

This implies that $\gamma_{0}=1$ since $\left|\gamma_{0}\right|=1$. Hence, by (47) we have

$$
S^{*}\left(\left.\varphi_{C(Y, \eta), y_{0}, 1}\right|_{E}\right)=\varphi_{C(X, \tau), x, 1},
$$

and so statement (I) holds.

Since $\varphi_{C(X, \tau), x, 1} \in \operatorname{Ext}\left(B_{C(X, \tau)^{*}}\right)$ for all $x$ in $X$, statement (I) implies that $Y_{0}$ is nonempty.

We next show that the following statement, namely, (II), holds.

(II) For each $y \in Y_{0}$ there exists a unique point $x \in X$ such that $(T f)(y)=f(x)$ for all $f$ in $C(X, \tau)$.

Let $y \in Y_{0}$. Then, $S^{*}\left(\left.\varphi_{C(Y, \eta), y, 1}\right|_{E}\right) \in \operatorname{Ext}\left(B_{C(X, \tau)^{*}}\right)$. Hence, by Theorem 1 , there exists $\left(x_{0}, \lambda_{0}\right) \in X \times \mathbb{S}_{\mathbb{C}}$ such that

$$
S^{*}\left(\left.\varphi_{C(Y, \eta), y, 1}\right|_{E}\right)=\varphi_{C(X, \tau), x_{0}, \lambda_{0}} .
$$

Since $1_{X} \in C(X, \tau)$ and $T 1_{X}=1_{Y}$, by (50) we have

$$
\begin{aligned}
\operatorname{Re}\left(\lambda_{0}\right) & =\operatorname{Re}\left(\lambda_{0} \cdot 1_{X}\left(x_{0}\right)\right)=\varphi_{C(X, \tau), x_{0}, \lambda_{0}}\left(1_{X}\right) \\
& =S^{*}\left(\left.\varphi_{C(Y, \eta), y, 1}\right|_{E}\right)\left(1_{X}\right)=\varphi_{C(Y, \eta), y, 1}\left(S 1_{X}\right) \\
& =\varphi_{C(Y, \eta), y, 1}\left(T 1_{X}\right)=\varphi_{C(Y, \eta), y, 1}\left(1_{Y}\right) \\
& =\operatorname{Re}\left(1 e_{C(Y, \eta), y}\left(1_{Y}\right)\right)=\operatorname{Re}\left(e_{C(Y, \eta), y}\left(1_{Y}\right)\right) \\
& =\operatorname{Re}\left(1_{Y}(y)\right)=\operatorname{Re}(1) \\
& =1 .
\end{aligned}
$$


This implies that $\lambda_{0}=1$ since $\left|\lambda_{0}\right|=1$. Thus, by (50) we have

$$
S^{*}\left(\left.\varphi_{C(Y, \eta), y, 1}\right|_{E}\right)=\varphi_{C(X, \tau), x_{0}, 1} .
$$

From (52) we give

$$
\operatorname{Re}((S f)(y))=\operatorname{Re}\left(f\left(x_{0}\right)\right), \quad \forall f \in C(X, \tau) .
$$

It follows that

$$
\operatorname{Re}((T f)(y))=\operatorname{Re}\left(f\left(x_{0}\right)\right), \quad \forall f \in C(X, \tau) .
$$

According to (54) and condition (ii), $E$ is extremely regular at $\{y, \eta(y)\}$. On the other hand, $C(X, \tau)$ is extremely regular at $\left\{x_{0}, \tau\left(x_{0}\right)\right\}$ by Lemma 4 . Hence, by Lemma 3, we deduce that $(S f)(y)=f\left(x_{0}\right)$ for all $f$ in $C(X, \tau)$ or $(S f)(y)=$ $f\left(\tau\left(x_{0}\right)\right)$ for all $f$ in $C(X, \tau)$. We assume that $x=x_{0}$ whenever $(S f)(y)=f\left(x_{0}\right)$ for all $f$ in $C(X, \tau)$ and $x=\tau\left(x_{0}\right)$ whenever $(S f)(y)=f\left(\tau\left(x_{0}\right)\right)$ for all $f$ in $C(X, \tau)$. Then, $x \in X$ and we have

$$
(T f)(y)=f(x), \quad \forall f \in C(X, \tau)
$$

This proves the existent of $x$. To show uniqueness, assume that there exists $x^{\prime} \in X$ such that

$$
(T f)(y)=f\left(x^{\prime}\right), \quad \forall f \in C(X, \tau) .
$$

From (55) and (56) we have $f(x)=f\left(x^{\prime}\right)$ for all $f$ in $C(X, \tau)$. This implies that $x=x^{\prime}$ since $C(X, \tau)$ separates the points of $X$. Thus, statement (II) holds.

Now we define the map $h: Y_{0} \rightarrow X$ by $h(y)=x$ whenever $(T f)(y)=f(x)$ for all $f$ in $C(X, \tau)$. The statement (II) implies that $h$ is well-defined. By definition of $h$, we have

$$
(T f)(y)=f(h(y)), \quad \forall f \in C(X, \tau) \forall y \in Y_{0} .
$$

We prove that $h \circ \eta=\tau \circ h$ in $Y_{0}$. Let $y \in Y_{0}$. For each $f \in$ $C(X, \tau)$, by (57) we have

$$
\begin{aligned}
f((h \circ \eta)(y)) & =f(h(\eta(y)))=(T f)(\eta(y)) \\
& =\overline{(T f)(y)}=\overline{f(h(y))} \\
& =f(\tau(h(y)))=f((\tau \circ h)(y)) .
\end{aligned}
$$

This implies that $(h \circ \eta)(y)=(\tau \circ h)(y)$ since $C(X, \tau)$ separates the points of $X$. Hence, $h \circ \eta=\tau \circ h$ on $Y_{0}$.
Continually, we show that $Y_{0}$ is a $\eta$-invariant. Let $y \in Y_{0}$. For each $f \in C(X, \tau)$ we have

$$
\begin{aligned}
S^{*}\left(\left.\varphi_{C(Y, \eta), \eta(y), 1}\right|_{E}\right)(f) & =\varphi_{C(Y, \eta), \eta(y), 1}(S f) \\
& =\varphi_{C(Y, \eta), \eta(y), 1}(T f) \\
& =\operatorname{Re}\left(1 e_{C(Y), \eta(y)), 1}(T f)\right) \\
& =\operatorname{Re}((T f)(\eta(y)))=\operatorname{Re}(\overline{(T f)(y)}) \\
& =\operatorname{Re}(\overline{(f(h(y)))}) \\
& =\operatorname{Re}(1 f(\tau(h(y)))) \\
& =\operatorname{Re}\left(\left.1 e_{C(X), \tau(h(y))}\right|_{C(X, \tau)}(f)\right) \\
& =\left(\operatorname{Re}\left(\left.1 e_{C(X), \tau(h(y))}\right|_{C(X, \tau)}\right)\right)(f) \\
& =\varphi_{C(X, \tau), \tau(h(y)), 1}(f) .
\end{aligned}
$$

This implies that

$$
S^{*}\left(\left.\varphi_{C(Y, \eta), \eta(y), 1}\right|_{E}\right)=\varphi_{C(X, \tau), \tau(h(y)), 1} .
$$

Hence, $S^{*}\left(\left.\varphi_{C(Y, \eta), y, 1}\right|_{E}\right) \in \operatorname{Ext}\left(B_{C(X, \tau)^{*}}\right)$ by Theorem 1 . Therefore, $\eta(y) \in Y_{0}$ and so $Y_{0}$ is $\eta$-invariant.

Now we show that $h$ is surjective. Let $x \in X$. According to statement (I), we deduce that there exists a point $y_{0} \in Y_{0}$ such that

$$
S^{*}\left(\varphi_{C(Y, \eta), y_{0}, 1}\right)=\varphi_{C(X, \tau), \tau(x), 1} .
$$

It follows that $y_{0} \in Y$ and we have

$$
\operatorname{Re}\left((T f)\left(y_{0}\right)\right)=\operatorname{Re}(f(x)), \quad \forall f \in C(X, \tau) .
$$

Statement (II) implies that there exists a point $x^{\prime} \in X$ such that

$$
(T f)\left(y_{0}\right)=f\left(x^{\prime}\right), \quad \forall f \in C(X, \tau) .
$$

From (62) and (63), we have $\operatorname{Re}(f(x))=\operatorname{Re}\left(f\left(x^{\prime}\right)\right)$ for all $f$ in $C(X, \tau)$. This implies that $x^{\prime} \in\{x, \tau(x)\}$ since $\operatorname{Re} C(X, \tau)$ separates the points of $X / \tau$. If $x^{\prime}=x$, then by (63) and definition of $h$ we have $x=h\left(y_{0}\right)$. If $x^{\prime}=\tau(x)$, then by (63) and definition of $h$ we have

$$
\begin{aligned}
(T f)\left(\eta\left(y_{0}\right)\right) & =\overline{(T f)\left(y_{0}\right)}=\overline{f\left(x^{\prime}\right)} \\
& =f\left(\tau\left(x^{\prime}\right)\right)=f(x),
\end{aligned}
$$

for all $f$ in $C(X, \tau)$, and so $x=h\left(\eta\left(y_{0}\right)\right)$. Therefore, $h$ is surjective.

Continually, we show that $Y_{0}$ is a boundary for $E$. Let $g \in$ $E$. Then, there exists a function $f \in C(X, \tau)$ such that $T f=$ g. Since $f$ is a continuous complex-valued function on the compact space $X$, there exists a point $x_{0} \in X$ such that $\|f\|_{X}=$ 
$\left|f\left(x_{0}\right)\right|$. The surjectivity of $h: Y_{0} \rightarrow X$ implies that there exists a point $y_{0} \in Y_{0}$ such that $h\left(y_{0}\right)=x_{0}$. Hence, $(T f)\left(y_{0}\right)=$ $f\left(x_{0}\right)$, and so

$$
\begin{aligned}
\|g\|_{Y} & =\|T f\|_{Y}=\|f\|_{X}=\left|f\left(x_{0}\right)\right| \\
& =\left|(T f)\left(y_{0}\right)\right|=\left|g\left(y_{0}\right)\right| .
\end{aligned}
$$

Therefore, $Y_{0}$ is a boundary for $E$.

We now check that $h: Y_{0} \rightarrow X$ is continuous. Let $y \in Y_{0}$, and let $\left\{y_{\alpha}\right\}_{\alpha \in J}$ be a net in $Y_{0}$ such that $\lim _{\alpha} y_{\alpha}=y$ in $Y_{0}$. Then, for each $f \in C(X, \tau)$ we have $\lim _{\alpha}(T f)\left(y_{\alpha}\right)=(T f)(y)$ in $X$. This implies that for each $\lim _{\alpha} f\left(h\left(y_{\alpha}\right)\right)=f(h(y))$ in $X$. Since $C(X, \tau)$ separates the points of $X$, we have $\lim _{\alpha} h\left(y_{\alpha}\right)=h(y)$ in $X$. Therefore, $h: Y_{0} \rightarrow X$ is continuous.

Finally, we show that $Y_{0}$ is closed in $Y$. Let $y \in \overline{Y_{0}}$, the closure of $Y_{0}$ in $Y$. Then, there exists a net $\left\{y_{\alpha}\right\}_{\alpha \in J}$ in $Y_{0}$ such that $\lim _{\alpha} y_{\alpha}=y$ in $Y$. Then, for each $f \in C(X, \tau)$ we have $\lim _{\alpha}(T f)\left(y_{\alpha}\right)=(T f)(y)$, and so $\lim _{\alpha} f\left(h\left(y_{\alpha}\right)\right)=(T f)(y)$ in $X$. Since the net $\left\{f\left(h\left(y_{\alpha}\right)\right)\right\}_{\alpha \in J}$ converges for all $f$ in $C(X, \tau)$, we conclude that the net $\left\{h\left(y_{\alpha}\right)\right\}_{\alpha \in J}$ converges in $X$ to a point $x \in X$. Hence, for each $f \in C(X, \tau)$ we have $\lim _{\alpha} f\left(h\left(y_{\alpha}\right)\right)=$ $f(x)$. Therefore, $(T f)(y)=f(h(y))$ for all $f$ in $C(X, \tau)$. This implies that for each $f \in C(X, \tau)$ we have

$$
\begin{aligned}
S^{*}\left(\left.\varphi_{C(Y, \eta), y, 1}\right|_{E}\right)(f) & =\left(\left.\varphi_{C(Y, \eta), y, 1}\right|_{E}\right)(S f) \\
& =\left(\varphi_{C(Y, \eta), y, 1}\right)(T f) \\
& =\operatorname{Re}\left(1 e_{C(Y), y}(T f)\right) \\
& =\operatorname{Re}((T f)(y)) \\
& =\operatorname{Re}(f(h(y))) \\
& =\operatorname{Re}\left(e_{C(X), h(y)}(f)\right) \\
& =\left(\operatorname{Re}\left(\left.1 e_{C(X), h(y)}\right|_{C(X, \tau)}\right)\right)(f) \\
& =\varphi_{C(X, \tau), h(y), 1}(f) .
\end{aligned}
$$

Hence,

$$
S^{*}\left(\left.\varphi_{C(Y, \eta), y, 1}\right|_{E}\right)=\varphi_{C(X, \tau), h(y), 1} .
$$

This implies that $S^{*}\left(\left.\varphi_{C(Y, \eta), y, 1}\right|_{E}\right) \in \operatorname{Ext}\left(B_{C(X, \tau)^{*}}\right)$, by Theorem 1. Therefore, $y \in Y_{0}$. So $Y_{0}$ is closed in $Y$.

Note that if $T: C(X, \tau) \rightarrow C(Y, \eta)$ is a linear isometry from $C(X, \tau)$ onto $C(Y, \eta)$, then $T(C(X, \tau))=C(Y, \eta)$ and so $T(C(X, \tau))$ is extremely regular at $\{y, \eta(y)\}$ for all $y$ in $Y$. Therefore, if $T: C(X, \tau) \rightarrow C(Y, \eta)$ is a linear isometry from $C(X, \tau)$ on $C(Y, \eta)$ with $T\left(1_{X}\right)=1_{Y}$, then conditions (i) and (ii) in Theorem 10 hold.

We now study the into case (not necessarily unitpreserving).

Theorem 11. Let $T: C(X, \tau) \rightarrow C(Y, \eta)$ be a linear isometry from $C(X, \tau)$ into $C(Y, \eta)$ satisfying the following conditions:

(i) $\left|T 1_{X}(y)\right|=1$ for all $y \in Y$, (ii) if $y \in Y$ and there is a point $x \in X$ such that $\operatorname{Re}\left(\overline{T 1_{X}(y)}(T f)(y)\right)=\operatorname{Re}(f(x))$ for all $f$ in $C(X, \tau)$, then $\left(\overline{T 1_{X}}\right) T(C(X, \tau))$ is extremely regular at $\{y, \tau(y)\}$.

Then, there exist a $\eta$-invariant closed boundary $Y_{0}$ for $T(C(X, \tau))$, a function $a \in C(Y, \eta)$ with $|a(y)|=1$ for all $y \in Y$, and a continuous map hrom $Y_{0}$ onto $X$ with $h \circ \eta=\tau \circ h$ on $Y_{0}$ such that

$$
(T f)(y)=a(y) f(h(y)), \quad \forall f \in C(X, \tau), \forall y \in Y_{0} .
$$

Proof. Assume that $a=T 1_{X}$. Then, $a \in C(Y, \eta)$, and by (i) we have $|a(y)|=1$ for all $y \in Y$. Clearly, $(\bar{a})(T f) \in C(Y, \eta)$ for all $C(Y, \eta)$, and $(\bar{a})\left(T 1_{X}\right)=1_{Y}$. We define the map $\bar{a} \cdot T$ : $C(X, \tau) \rightarrow C(Y, \eta)$ by $(\bar{a} \cdot T)(f)=(\bar{a})(T f)$. It is easy to see that $\bar{a} \cdot T$ is a linear isometry from $C(X, \tau)$ into $C(Y, \eta)$ such that $(\bar{a} \cdot T)\left(1_{X}\right)=1_{Y}$.

Applying (ii), we can easily show that if $y \in Y$ and there exists a point $x \in X$ such that $\operatorname{Re}(((\bar{a} \cdot T) f)(y))=\operatorname{Re}(f(x))$ for all $f$ in $C(X, \tau)$, then $(\bar{a} \cdot T)(C(X, \tau))$ is extremely regular at $\{y, \eta(y)\}$. Let $E=T(C(X, \tau))$. Then, $\bar{a} E=(\bar{a} \cdot T)(C(X, \tau))$. We define $S: C(X, \tau) \rightarrow E$ by $S f=T f$ and $S_{1}: C(X, \tau) \rightarrow \bar{a} E$ by $S_{1} f=(\bar{a} \cdot T) f$. Clearly, $S$ is a linear isometry from $C(X, \tau)$ onto $E$, and $S_{1}$ is a linear isometry from $C(X, \tau)$ onto $\bar{a} E$. Now, we define

$$
Y_{0}=\left\{y \in Y: S^{*}\left(\left.\varphi_{C(Y, \eta), y, \overline{a(y)}}\right|_{E}\right) \in \operatorname{Ext}\left(B_{C(X, \tau)^{*}}\right)\right\} .
$$

It is easy to see that

$$
Y_{0}=\left\{y \in Y: S_{1}^{*}\left(\left.\varphi_{C(Y, \eta), y, 1}\right|_{\bar{a} E}\right) \in \operatorname{Ext}\left(B_{C(X, \tau)^{*}}\right)\right\} .
$$

By given arguments in the proof of Theorem 10, $Y_{0}$ is a $\eta$-invariant closed boundary for $\bar{a} E$, and there exists a continuous function $h$ from $Y_{0}$ onto $X$ with $h \circ \eta=\tau \circ h$ such that

$$
((\bar{a} \cdot T)(f))(y)=f(h(y)), \quad \forall f \in C(X, \tau), \forall y \in Y_{0} .
$$

We now show that $Y_{0}$ is a boundary for $E$. Let $g \in E$. Then, $\bar{a} g \in \bar{a} E$. Hence, there exists a point $y_{0} \in Y_{0}$ such that

$$
\|\bar{a} g\|_{Y}=\left|(\bar{a} g)\left(y_{0}\right)\right| \text {. }
$$

Since $|a(y)|=1$ for all $y \in Y$, we conclude that

$$
\|\bar{a} g\|_{Y}=\|g\|_{Y} .
$$

From (72) and (73), we have

$$
\begin{aligned}
\|g\|_{Y} & =\left|\bar{a} g\left(y_{0}\right)\right|=\left|\bar{a}\left(y_{0}\right) g\left(y_{0}\right)\right|=\left|\overline{a\left(y_{0}\right)} g\left(y_{0}\right)\right| \\
& =\left|\overline{a\left(y_{0}\right)}\right|\left|g\left(y_{0}\right)\right|=\left|a\left(y_{0}\right)\right|\left|g\left(y_{0}\right)\right|=\left|g\left(y_{0}\right)\right| .
\end{aligned}
$$

Therefore, $Y_{0}$ is a boundary for $E$.

Finally, we show that

$$
(T f)(y)=a(y) f(h(y)), \quad \forall f \in C(X, \tau), \forall y \in Y_{0} .
$$


Let $f \in C(X, \tau)$ and $y \in Y_{0}$. From (73), we have

$$
((\bar{a} \cdot T)(f))(y)=f(h(y))
$$

Applying (76), we deduce that

$$
\begin{aligned}
(T f)(y) & =a(y) \overline{a\left(y_{0}\right)}(T f)(y)=a(y)((\bar{a})(T f))(y) \\
& =a(y)((\bar{a} \cdot T) f)(y)=a(y) f(h(y)) .
\end{aligned}
$$

Hence, the proof is complete.

Note that if $T: C(X, \tau) \rightarrow C(Y, \eta)$ is a linear isometry from $C(X, \tau)$ onto $C(Y, \eta)$, then $\left|T 1_{x}(y)\right|=1$ for all $y$ in $Y$ by Lemma 8 and $T(C(X, \tau))$ is extremely regular at $\{y, \eta(y)\}$ for all $y$ in $Y$ by Lemma 4, and so the conditions (i) and (ii) in Theorem 11 hold.

The following result shows that the triple $\left\{Y_{0}, a, \eta\right\}$ associated to isometry $T$ in Theorem 11 possesses a universal property.

Corollary 12. Let $T: C(X, \tau) \rightarrow C(Y, \eta)$ be a linear isometry from $C(X, \tau)$ into $C(Y, \eta)$ satisfying the following conditions:

(i) $\left|T 1_{X}(y)\right|=1$ for all $y \in Y$,

(ii) if $y \in Y$ and there is a point $x \in X$ such that $\operatorname{Re}\left(\overline{T 1_{X}(y)}(T f)(y)\right)=\operatorname{Re}(f(x))$ for all $f$ in $C(X, \tau)$, then $T(C(X, \tau))$ is extremely regular at $\{y, \tau(y)\}$.

Let $Y_{0}$ and $h$ be as in Theorem 11. If $Y_{1}$ is a nonempty $\eta$ invariant subset (not necessarily closed) of $Y$ and $b \in C(Y, \eta)$ with $|b(y)|=1$ for all $y \in Y_{1}$ and $k: Y_{1} \rightarrow X$ is a continuous map (not necessarily onto) with $h \circ \eta=\tau \circ k$ on $Y_{1}$ such that

$$
(T f)(y)=b(y) f(k(y)), \quad \forall f \in C(X, \tau), \forall y \in Y_{1} \text {, }
$$

then $Y_{1} \subseteq Y_{0}, b=\left.a\right|_{Y_{1}}$ and $k=\left.h\right|_{Y_{1}}$.

Proof. Let $y \in Y_{1}$. Since $1_{X} \in C(X, \tau)$, taking $f=1_{X}$ in the above expression of $T f$, we have $b(y)=\left(T 1_{X}\right)(y)=a(y)$. Thus, $b=\left.a\right|_{Y_{1}}$.

Let $E=T(C(X, \tau))$ and $S: C(X, \tau) \rightarrow E$ defined by $S f=T f$. Then,

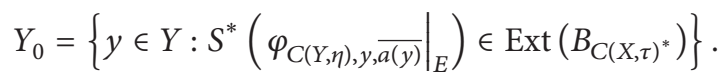

Let $y \in Y_{1}$. Then, the above expression of $T f$ reads as follows:

$$
(T f)(y)=b(y) f(k(y)), \quad \forall f \in C(X, \tau) .
$$

From (80), for each $f \in C(X, \tau)$ we have

$$
\begin{aligned}
S^{*}\left(\left.\varphi_{C(Y, \eta), y, \overline{a(y)}}\right|_{E}\right)(f) & =\left(\left.\varphi_{C(Y, \eta), y, \overline{a(y)}}\right|_{E}\right)(T f) \\
& =\operatorname{Re}\left(\overline{a(y)} e_{C(Y), y}(T f)\right) \\
& =\operatorname{Re}(\overline{a(y)}(T f)(y)) \\
& =\operatorname{Re}(\overline{a(y)} a(y) f(k(y))) \\
& =\operatorname{Re}\left(|a(y)|^{2} f(k(y))\right) \\
& =\operatorname{Re}(f(k(y))) \\
& =\left(\operatorname{Re}\left(1 e_{C(X), k(y)}\right)\right)(f) \\
& =\varphi_{C(X, \tau), k(y), 1}(f) .
\end{aligned}
$$

This implies that

$$
S^{*}\left(\left.\varphi_{C(Y, \eta), y, \overline{a(y)}}\right|_{E}\right)=\varphi_{C(X, \tau), k(y), 1} .
$$

From (82), we deduce that $S^{*}\left(\left.\varphi_{C(Y, \eta), y, \overline{a(y)}}\right|_{E}\right)$ is an extreme point of Ext $B_{C(X, \tau)}$ by Theorem 1 . Therefore, $y \in Y_{0}$. So $Y_{1} \subseteq Y_{0}$.

We now show that $k=\left.h\right|_{Y_{1}}$. Let $y \in Y_{1}$. By (80), we have

$$
(T f)(y)=a(y) f(k(y)), \quad \forall f \in C(X, \tau) .
$$

Since $Y_{1} \subseteq Y_{0}$, we deduce that $y \in Y_{0}$, and so by Theorem 11 we have

$$
(T f)(y)=a(y) f(h(y)), \quad \forall f \in C(X, \tau) .
$$

Since $|a(y)|=1$, by (83) and (84) we have

$$
f(k(y))=f(h(y)), \quad \forall f \in C(X, \tau) .
$$

This implies that $k(y)=h(y)$ since $C(X, \tau)$ separates the points of $X$. Thus, $k=\left.h\right|_{Y_{1}}$. Hence, the proof is complete.

\section{Acknowledgment}

The authors would like to thank the referee for some valuable and useful comments.

\section{References}

[1] S. H. Kulkarni and B. V. Limaye, "Extreme points of the unit ball in the dual spaces of some real subspaces of $C(X)$," Glasnik Matematički, vol. 29, no. 49, pp. 333-340, 1994.

[2] N. Dunford and J. T. Schwartz, Linear Operators, Part 1, General Theory, Interscience, New York, NY, USA, 1958.

[3] S. H. Kulkarni and B. V. Limaye, Real Function Algebras, Marcel Dekker, New York, NY, USA, 1992.

[4] S. H. Kulkarni and B. V. Limaye, "Gleason parts of real function algebras," Canadian Journal of Mathematics, vol. 33, no. 1, pp. 181-200, 1981. 
[5] W. Holsztyński, "Continuous mappings induced by isometries of spaces of continuous function," Studia Mathematica, vol. 26, pp. 133-136, 1966.

[6] W. Novinger, "Linear isometries of subspaces of continuous functions," Studia Mathematica, vol. 53, pp. 273-276, 1975.

[7] S. H. Kulkarni and S. Arundhathi, "Isometries of real function algebras," Commentationes Mathematicae, vol. 30, no. 2, pp. 343-356, 1991.

[8] M. Cambern, "A Holsztynski theorem for spaces of continuous vector-valued functions," Studia Mathematica, vol. 63, no. 3, pp. 213-217, 1978.

[9] J.-S. Jeang and N.-C. Wong, "Weighted composition operators of $C_{0}(X)^{\prime}$," Journal of Mathematical Analysis and Applications, vol. 201, no. 3, pp. 981-993, 1996.

[10] J. Araujo and J. J. Font, "Linear isometries between subspaces of continuous functions," Transactions of the American Mathematical Society, vol. 349, no. 1, pp. 413-428, 1997.

[11] A. Jiménez-Vargas and M. Villegas-Vallecillos, "Into linear isometries between spaces of Lipschitz functions," Houston Journal of Mathematics, vol. 34, no. 4, pp. 1165-1184, 2008.

[12] S. Mazur and S. Ulam, "Sur les transformations isometriques d'espace vectoriels normes," Comptes Rendus de l'Académie des Sciences, vol. 194, pp. 946-948, 1932. 


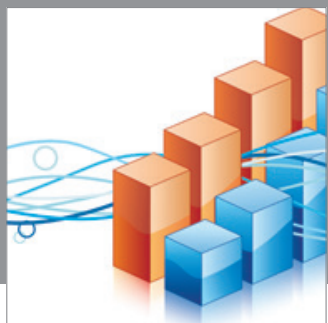

Advances in

Operations Research

mansans

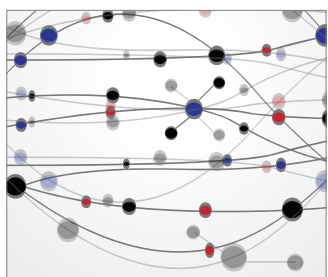

The Scientific World Journal
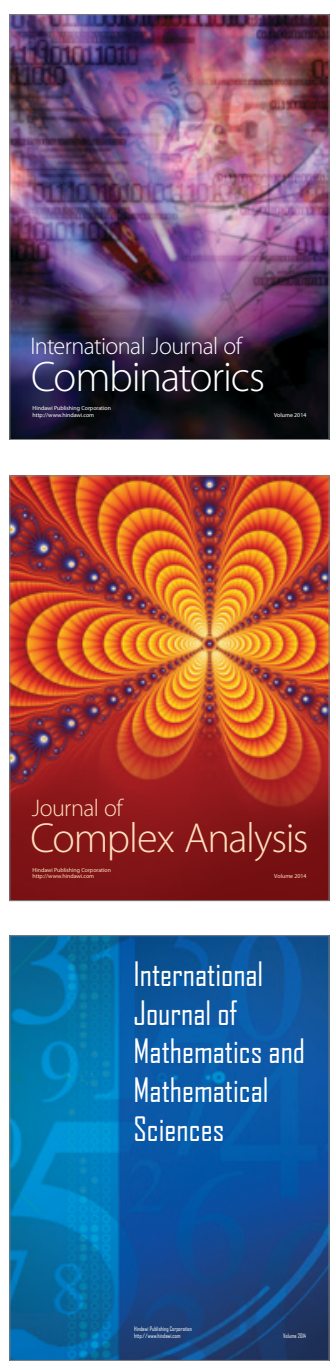
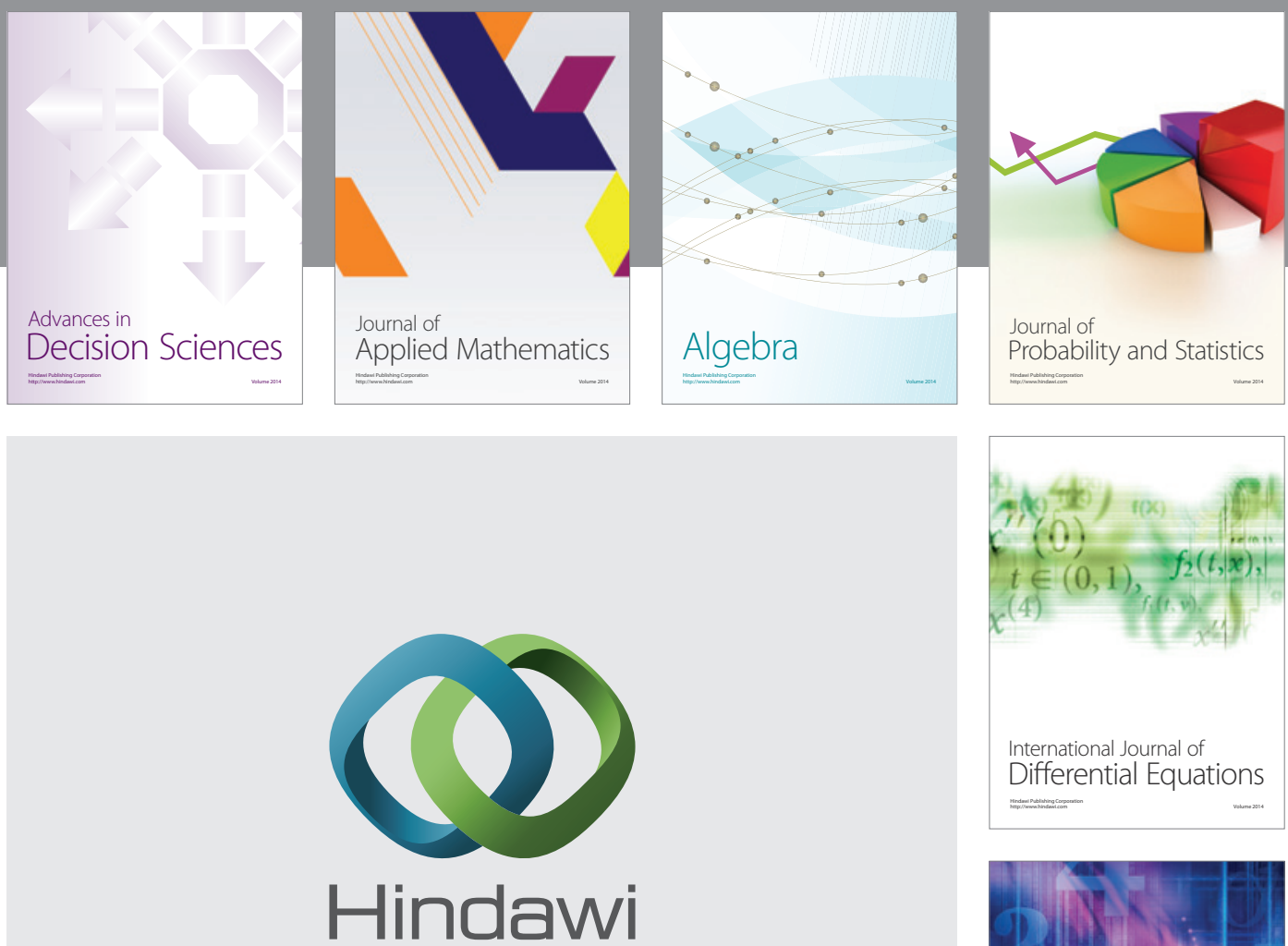

Submit your manuscripts at http://www.hindawi.com
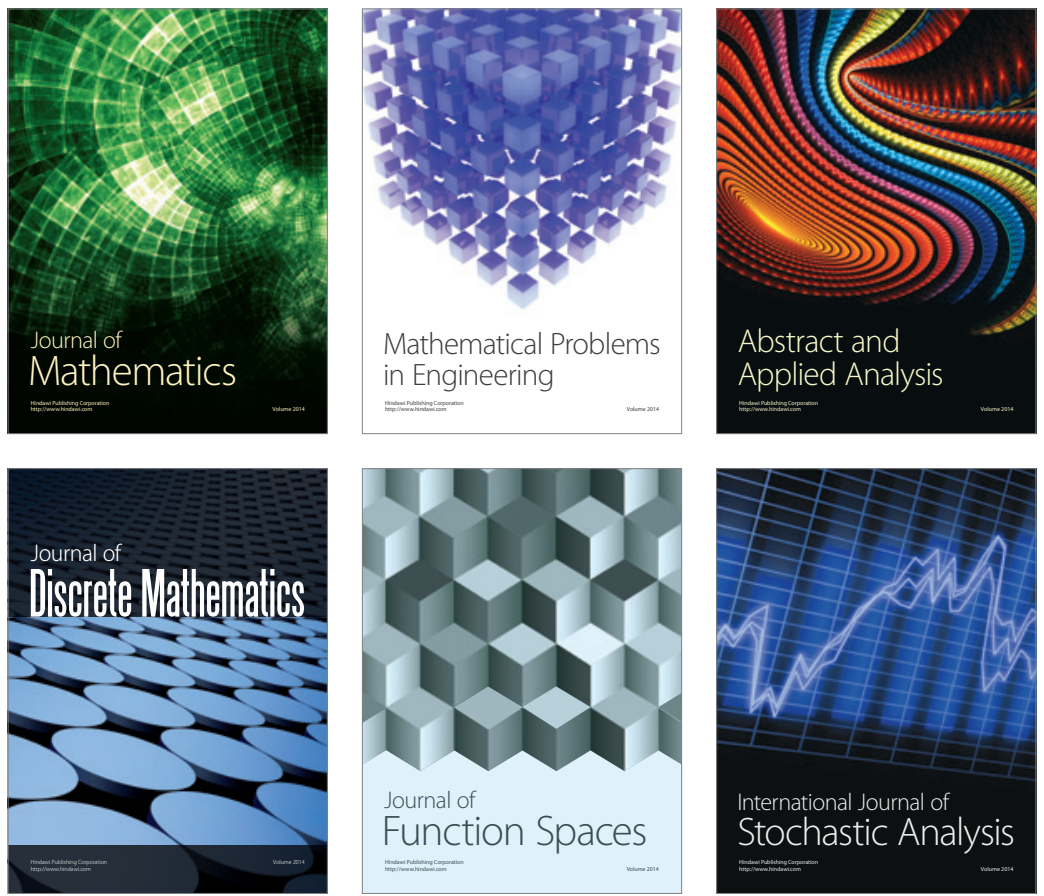

Journal of

Function Spaces

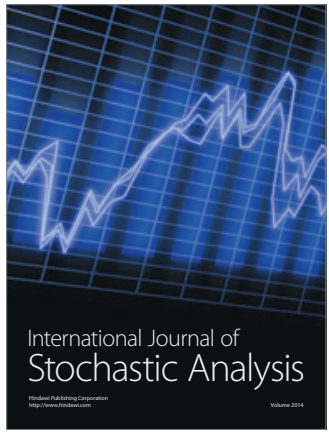

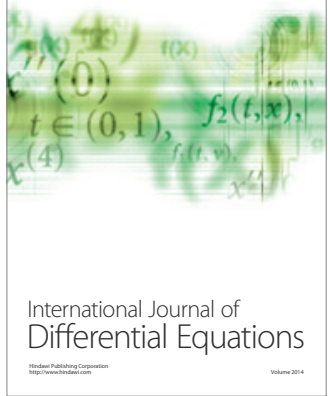
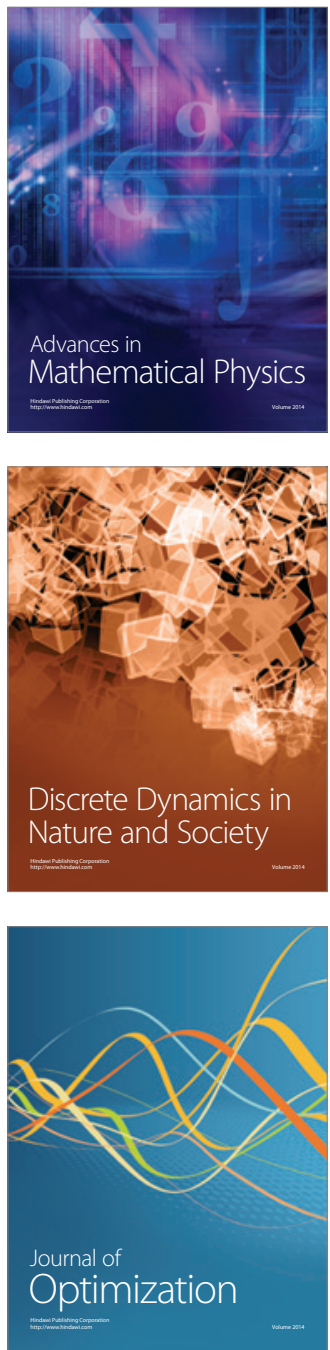